\title{
Repensando acesso, qualificando o cuidado: relato do trabalho em duas equipes de saúde da família
}

\author{
Rethinking access, qualifying care: a report of the work in two family health care teams \\ Repensar el acceso, mejorar la atención: un informe del trabajo en dos equipos de atención primaria
}

Carolina Lopes de Lima Reigada ${ }^{a}$

\section{Resumo}

Em março de 2015, a residência em medicina de família e comunidade foi incorporada a duas equipes de um centro de saúde: uma com população vulnerável e grande rotatividade de médicos, e outra com população principalmente da classe média. Após três meses, percebemos que os profissionais estavam sobrecarregados na maioria dos turnos de atendimento, não tinham horário de almoço e saíam da unidade após o fechamento. Na avaliação $360^{\circ}$ pela preceptora, os pacientes aprovavam o atendimento, mas reclamavam do tempo de espera para a consulta. $O$ objetivo foi reavaliar o processo de trabalho e melhorar a satisfação dos pacientes e profissionais. Para tal, levantamos os nós críticos nas agendas diárias e adaptamos a capacidade das agendas às necessidades das populações, e optamos pelo acesso avançado, além de outros acordos entre a equipe para organizar a demanda. Toda a equipe participou do processo de planejamento e incorporação das ações. Logo nas primeiras duas semanas, os profissionais perceberam diminuição na sobrecarga de trabalho. Nossos próximos passos são aumentar participação popular e melhorar vigilância sobre a população, evitando a lei de cuidados inversos.

\begin{abstract}
In March 2015, the residence in family medicine was incorporated into two teams at a health center: one which has existed for eight years, attends a vulnerable population and has a high turnover of doctors, and another with a population mostly from middle class. After three months, we observed that professionals were overwhelmed in most service shifts, had no lunch break, and left after closing time. At the 360 review conducted by the preceptor, patients approved the consultations with residents, but complained of the waiting time. Our objective was to re-evaluate the work process and improve the satisfaction of patients and professionals. To this end, we studied each team, determined the key critical nodes in daily schedules, adapted the capacity of the teams to the needs of the populations, and opted for advanced access, in addition to other agreements between the staff to organize and optimize service. The whole team participated in planning and implementing these actions. During the first two weeks, practitioners were able to take their lunch breaks, leaving the center on time, and there was an increase in satisfaction. For our next step, we intend to raise popular participation and avoid the inverse care law by searching actively for patients who do not attend the clinic.
\end{abstract}

\section{Resumen}

En marzo del 2015, la residencia en medicina familiar se ha unido a dos equipos de un centro de salud: uno equipo con una población vulnerable y una alta rotación de médicos, e otro con una población mayoritariamente de la clase media. Después de tres meses, nos dimos cuenta de que los profesionales estaban sobrecargados en la mayoría de los turnos de servicio, sin la hora del almuerzo y dejaban la clínica después del cierre. En la evaluación de $360^{\circ}$ por la preceptora, los pacientes aprobaron la atención médica, pero se quejaron del tiempo de espera para la consulta. El objetivo era volver a evaluar el proceso de trabajo y mejorar la satisfacción de los pacientes y de los profesionales. Para ello, analizamos los nodos críticos en los horarios diarios para adaptar la capacidad de los programas a las necesidades de la población, y optamos por el acceso avanzado, además de otros acuerdos entre el personal para organizar la demanda. Todo el equipo participó en la planificación y el proceso de incorporación de acciones. Durante las dos primeras semanas, los médicos se dieron cuenta de la disminución de la carga de trabajo. Nuestros próximos pasos son aumentar la participación popular y mejorar la vigilancia de la población, evitando la ley de atención inversa.

Como citar: Reigada CLL. Repensando acesso, qualificando o cuidado: relato do trabalho em duas equipes de saúde da família. Rev Bras Med Fam Comunidade. 2017;12(39):1-7. http://dx.doi.org/10.5712/rbmfc12(39)1451
Palavras-chave:

Estratégia Saúde da Família Acesso aos Serviços de Saúde Assistência Integral à Saúde

Keywords:

Family Health Strategy

Health Services Accessibility Comprehensive Health Care

Palabras clave:

Estrategia de Salud Familiar Accesibilidad a los

Servicios de Salud

Atención Integral de Salud

Fonte de financiamento:

declaram não haver.

Parecer CEP:

não se aplica.

Conflito de interesses:

declaram não haver.

Procedência e revisão por pares: revisado por pares.

Recebido em: 18/01/2017.

Aprovado em: 23/04/2017. 


\section{Introdução}

Universalidade é uma diretriz do SUS, garantida por Lei, relacionada ao acesso da população aos serviços de saúde. Entretanto, a legalidade de uma proposta não assegura sua implementação. ${ }^{1}$

Em 2006, a Estratégia Saúde da Família (ESF) foi consolidada como estratégia para efetivação da atenção primária à saúde (APS). ${ }^{2,3}$ A ESF busca aproximar-se da população, com visão ampliada do processo saúde-doença, provendo assistência efetiva, adaptada às necessidades locais, priorizando a produção compartilhada de saúde. ${ }^{2}$ Para tal, é necessário que os sujeitos tenham acesso facilitado a sua equipe de saúde, decidindo quanto ao momento em que se dará o encontro com os profissionais. Um acesso facilitado é uma das prerrogativas para efetivação de planos terapêuticos centrados no usuário.

Apesar de crucial para a ESF, não há tecnologias organizacionais consolidadas referentes à dimensão do acesso. ${ }^{4}$ A diretriz do acolhimento ${ }^{5}$ ressalta a importância da clínica ampliada e responsabilização da equipe pelo usuário, mas não estabelece metas de atendimento, tempo de espera aceitável para consulta ou formas de avaliação do acesso provido. ${ }^{4}$ Muitas formas de avaliação da qualidade da equipe de saúde da família têm enfoques abrangentes, mas não avaliam detalhadamente o acesso. ${ }^{4}$

Há poucos estudos abordando tempo de espera ou outras facetas do acesso na ESF. De fato, quando avaliado por usuários e profissionais de diferentes regiões brasileiras, é considerado uma fragilidade dos serviços, como em São Luís (MA), ${ }^{6}$ Petrópolis (RJ), ${ }^{7}$ Curitiba (PR), ${ }^{8}$ Porto Alegre (RS), ${ }^{9}$ Goiás e Mato Grosso do Sul, ${ }^{10}$ Florianópolis (SC), ${ }^{11}$ Macaíba (RN) ${ }^{12}$ e no próprio Rio de Janeiro (RJ), ${ }^{13}$ seja pelo tempo de espera, horário de funcionamento das unidades ou outros fatores.

Acesso é um conceito polissêmico: pode ser entendido como ato de chegar e ingressar no serviço ou indicar o grau de facilidade para obter cuidados de saúde, de maneira conveniente. ${ }^{2,3,14-16}$ Tais abordagens trazem a perspectiva do usuário como crucial para determinar o momento em que o acesso é realizado, não sendo prerrogativa somente do serviço de saúde.

O Centro de Saúde 1 (nome fictício) foi inaugurado em dezembro de 2014, contando com quatro equipes de saúde da família. Em duas delas, foi incorporada a residência em Medicina de Família e Comunidade (MFC).

Uma dessas, equipe Azul (nome fictício), existia há oito anos, em outra localidade. Atende a uma população vulnerável e, nos últimos anos, vivenciou alta rotatividade de médicos. A outra, equipe Verde (nome fictício), foi inaugurada em março de 2015, responsabilizando-se sobre uma população principalmente da classe média.

Durante os primeiros meses da residência, foi notória a sobrecarga de trabalho dos profissionais dessas equipes. Além de verbalizar seu cansaço, residentes e enfermeiros não tinham horário de almoço e era comum continuarem atendendo aos pacientes após o horário de fechamento da unidade, principalmente de segunda a quarta-feira. Às quintas e sextas-feiras, alguns horários ficavam ociosos.

Somada a essa insatisfação dos profissionais, a preceptora aplicou uma avaliação $360^{\circ 17}$ ao fim do primeiro trimestre e percebeu que, apesar da unanimidade dos pacientes aprovar o atendimento pelas residentes, todos apontaram o tempo de espera na unidade como ponto negativo, que implicava na satisfação com o serviço. 
Por fim, notamos que os mesmos pacientes se repetiam nas consultas ao longo das semanas, com pouca resolutividade no cuidado.

Sendo assim, foi desenvolvida a seguinte intervenção, com o objetivo de reavaliar o processo de trabalho das equipes e aumentar a satisfação de pacientes e profissionais.

\section{Desenvolvimento}

A preceptora organizou uma reunião com os profissionais das duas equipes para levantar os principais nós críticos ${ }^{18}$ nas agendas de médicos e enfermeiros. Além dos problemas acima, foram apontados: dificuldade em acessar a equipe de saúde perpetua a lei de cuidados inversos ${ }^{19}$ e aumenta a chance de que o paciente desenvolva complicações que poderiam ter sido evitadas, ou procure serviços de emergência como porta de entrada no sistema de saúde.

A metodologia utilizada foi brainstorming,$^{20}$ seguida de enumeração por consenso das seguintes demandas que, segundo os profissionais, contribuem para a lotação das agendas: a) solicitação de atestados para atividade física; b) solicitação de liberação para retornar à creche/escola após período de afastamento por atestado médico; c) retorno de pacientes com doenças crônicas não degenerativas para mostrar exames laboratoriais normais. Outro ponto levantado foi a necessidade de maior agilidade para atendimento às gestantes e puérperas.

Para tais problemas, foram propostas as seguintes soluções, imediatamente implementadas: a) oferecer, semanalmente, horário para avaliação e liberação de atestados de saúde; b) pacientes para liberação de retorno à creche/escola, consultas de pré-natal e puericultura serão colocados na agenda do profissional em atendimento no momento, mas logo encaminhados ao técnico de enfermagem para avaliação de sinais vitais; c) resultados laboratoriais normais serão anotados nos prontuários e esses pacientes serão encaminhados ao grupo semanal de promoção de saúde da unidade; d) resultados de colpocitológico e mamografia normais serão anotados no prontuário, e essas pacientes serão encaminhadas ao grupo semanal de mulheres da unidade. Nos dois últimos casos, o retorno à consulta se fará de acordo com a necessidade do paciente, não somente para mostrar exames.

Outros acordos foram: todos iniciarão o atendimento pontualmente às 8 e às 13 horas; as consultas deverão ser as mais resolutivas possíveis, obedecendo ao intervalo recomendado de retorno e individualização dos casos, evitando marcar consultas desnecessárias; o mês de retorno do paciente deve ser indicado de forma clara na receita e prontuário.

Outro problema levantado foi a demora para encontrar resultados de exames laboratoriais durante as consultas, alongando seu tempo de duração. Por isso, também acordamos que os agentes comunitários de saúde (ACS) deveriam separar resultados de exames laboratoriais dos pacientes a serem consultados. Infelizmente, esse acordo não foi sedimentado na prática.

Havia, ainda, a preocupação em adaptar a agenda das equipes à população atendida. Nesse sentido, outros problemas foram apontados: as agendas das equipes tinham turnos "fechados", em que não se podia encaixar demanda livre (ou externa), pois eram dedicados exclusivamente à coleta de preventivo ou interconsultas (matriciamento); e não era priorizado o acolhimento dos pacientes pela própria equipe. 
De forma a organizar a agenda das equipes, a preceptora fez um estudo da população de cada equipe, chegando ao resultado descrito nas Tabelas 1 e 2.

Tabela 1. Total de consultas necessárias para atendimento à população da equipe Verde.

\begin{tabular}{lcc}
\hline Equipe Verde & População & Quantidade de consultas/ano \\
\hline Mulheres 25 a 64 anos & 659 & 219 \\
Pessoas com DM & 104 & 312 \\
Pessoas com HAS & 353 & 1059 \\
Crianças até 2 anos & 45 & 450 \\
Gestantes & 22 & 220 \\
Pessoas tratando TB & 5 & 35 \\
\hline
\end{tabular}

DM: Diabetes Mellitus; HAS: Hipertensão Arterial Sistêmica; TB: Tuberculose.

Gerando um total de 2295 consultas ao ano. Em um ano, sendo 253 dias úteis, médico e enfermeiro devem atender um total de 9 pessoas por dia. Considerando 20 pacientes em demanda externa (média da equipe), o total seria de 29 atendimentos ao dia.

Tabela 2. Total de consultas necessárias para atendimento à população da equipe Azul.

\begin{tabular}{lcc}
\hline Equipe Azul & População & Quantidade de consultas/ano \\
\hline Mulheres de 25 a 64 anos & 674 & 224 \\
Pessoas com DM & 75 & 225 \\
Pessoas com HAS & 365 & 1095 \\
Crianças até 2 anos & 97 & 970 \\
Gestantes & 45 & 450 \\
Pessoas tratando TB & 7 & 56 \\
\hline DM:Diabetes Mellitus: HAS:Hipertensão Arterial Sistêmica: TB: Tubrculose.
\end{tabular}

DM: Diabetes Mellitus; HAS: Hipertensão Arterial Sistêmica; TB: Tuberculose.

Gerando um total de 3020 consultas ao ano. Em 253 dias úteis, médico e enfermeiro devem atender 12 pessoas por dia. Considerando 30 pacientes em demanda externa (média da equipe), o total seria de 42 atendimentos por dia.

As agendas foram então adaptadas à quantidade necessária de atendimentos. Após discussão entre os profissionais e a gerente da unidade, a mesma nos deu anuência para instituir o acesso avançado, ${ }^{21-23}$ ou agendamento aberto, por um período de experiência. A ressalva imposta foi que, no caso do paciente não conseguir atendimento para o mesmo dia, ele sairia da unidade com o retorno marcado, seja em consulta ou grupo de saúde. A gerência não permitiu mudar a forma de agendamento dos matriciamentos, porém concordou com a realização dos grupos de saúde.

Uma regra que se tornou importante é que o ACS responsável pela recepção é o único que pode incluir pacientes na agenda do médico ou enfermeiro de sua equipe. Muitas vezes, enfermeiros de outras equipes atendiam a um paciente e, ao invés de pedir interconsulta para resolução do caso, incluíam o paciente na agenda do médico, à frente de outros que estavam esperando há mais tempo, gerando desconforto. Nesses casos, as interconsultas foram estimuladas, otimizando o tempo dos profissionais e pacientes - que não precisavam repetir sua história a um novo profissional - e melhorando a relação entre os profissionais, estimulando a interdisciplinaridade. 
Apoiando a transição, a preceptora ficou com os ACS durante as primeiras duas semanas de implantação do novo processo de trabalho, e foram elaborados conjuntamente: a) fluxogramas indicando as condutas a serem tomadas no acolhimento ao paciente, em várias situações; b) folhetos informativos que ficaram disponíveis na recepção: o primeiro, "Conheça sua equipe de saúde da família", com nomes e horários de atendimentos de todos os profissionais das equipes; o segundo, "Venha participar dos nossos grupos!", listando todos os grupos disponíveis na unidade, horários e responsáveis.

\section{Resultados e Conclusão}

Na primeira semana, notamos diminuição da sobrecarga dos profissionais e, segundo os mesmos, uma melhora na qualidade do atendimento. Um relato espontâneo de uma das residentes foi: "Não sei que magia você fez com a agenda, mas eu nunca atendi tão focada no paciente que estou consultando. Antes eu não conseguia me concentrar, com tanta gente me esperando e batendo na porta".

Objetivamente, conseguimos terminar o turno da manhã entre 12 e 12:20 horas e o turno da tarde sempre às 17 horas, preservando os horários de almoço e de fechamento da unidade.

As duas primeiras semanas foram de sobrecarga para os ACS e a preceptora, no acolhimento à população. Resolvemos todas as demandas dos pacientes que procuraram as equipes, porém nem todos foram encaminhados a consultas médicas, por isso, alguns pacientes demonstraram insatisfação. Porém, a partir da terceira semana, os ACS passaram a avaliar as mudanças como positivas, apontando como vantagens: poder dizer aos pacientes o horário aproximado em que serão atendidos; exercer a escuta na recepção e aumentar sua resolutividade; diminuição da reclamação dos pacientes quanto ao tempo de espera.

Julgamos que tivemos sucesso, principalmente ao diminuir a sobrecarga dos profissionais e tornar os pacientes mais cientes da organização do trabalho e mais participativos em seu próprio cuidado.

Como fortalezas, apontamos a construção coletiva, com participação de toda a equipe no diagnóstico de problemas e proposição de soluções, o que legitimou as mudanças e motivou os profissionais, que tiveram maior gestão de seu processo de trabalho. A presença da preceptora da residência em MFC na supervisão do trabalho das equipes foi crucial para o trabalho de avaliação e intervenção, destacando a importância da residência na formação e qualificação dos profissionais atuando na ESF e da valorização do trabalho do preceptor nesse momento de expansão e consolidação da APS.

Como fragilidades, apontamos a necessidade de melhorar a vigilância sobre o território, pois muitos pacientes nunca foram vistos pelas equipes. Nossa estratégia foi levantar e listar todas as pessoas que não foram consultadas no último ano. Essas listas foram entregues aos ACS, para busca ativa. Estamos no início desse trabalho, mas identificamos muitos pacientes que se mudaram da área e ainda estavam com cadastro ativo e pacientes (principalmente homens) que trabalham durante o horário de funcionamento da unidade.

Outra fragilidade foi a pouca participação popular nas decisões sobre a organização do trabalho. Para aumentá-la, concordamos que a primeira reunião de equipe de cada mês será aberta à população e acontecerá em local próximo à residência das pessoas, divulgada previamente no centro de saúde, durante as consultas e visitas domiciliares. Além disso, pretendemos perguntar aos pacientes sobre sua percepção do acesso à sua equipe de referência, utilizando o questionário Primary Care Assessment Tool (PCA-Tool). Uma desvantagem já levantada foi o fato da unidade não abrir fora do horário comercial nem aos fins de semana. 
A organização da equipe para atender às necessidades específicas de sua comunidade, inclusive em relação ao processo de trabalho, é essencial para prover acesso real aos pacientes. Acesso também depende da adaptação da equipe às necessidades e funcionamento da população à qual serve.

\section{Referências}

1. Assis MMA, Villa TCS, Nascimento MAA. Acesso aos serviços de saúde: uma possibilidade a ser construída na prática. Ciênc Saúde Coletiva. 2003;8(3):815-23. DOI: http://dx.doi.org/10.1590/S1413-81232003000300016

2. Passamani JD, Silva MZ, Araújo MD. Estratégia Saúde da Família: o acesso em um território de classe média. UFES Rev Odontol. 2008;10(2):37-45.

3. Norman AH, Tesser CD. Acesso ao cuidado na Estratégia Saúde da Família: equilíbrio entre demanda espontânea e prevenção/promoção da saúde. Saude Soc. 2015;24(1):165-79. DOI:http://dx.doi.org/10.1590/S0104-12902015000100013

4. Tesser CD, Norman AH. Repensando o acesso ao cuidado na Estratégia Saúde da Família. Saúde Soc.2014;23(3):869-83.

5. Brasil. Ministério da Saúde. Secretaria-Executiva. Núcleo Técnico da Política Nacional de Humanização. HumanizaSUS Política Nacional de Humanização: A humanização como Eixo Norteador das Práticas de Atenção e Gestão em Todas as Instâncias do SUS. Internet. Brasília: Ministério da Saúde; 2004, 20 p. Série B. Textos básicos de saúde. [acesso 2016 Maio 3]. Disponível em: http://bvsms.saude.gov.br/bvs/publicacoes/humanizasus_2004.pdf

6. Reis RS, Coimbra LC, Silva AM, Santos AM, Britto e Alves MTSS, Lamy ZC, et al. Acesso e utilização dos serviços na Estratégia Saúde da Família na perspectiva dos gestores, profissionais e usuários. Ciênc Saúde Coletiva. 2013;18(11):332131. DOI: http://dx.doi.org/10.1590/S1413-81232013001100022

7. Macinko J, Almeida C, dos SE, de Sá PK. Organization and delivery of primary health care services in Petrópolis, Brazil. Int J Health Plann Manage. 2004;19(4):303-17. DOI: http://dx.doi.org/10.1002/hpm.766

8. Chomatas E, Vigo A, Marty I, Hauser L, Harzheim E. Avaliação da presença e extensão dos atributos da atenção primária em Curitiba. Rev Bras Med Fam Comunidade. 2013;8(29):294 303. http://dx.doi.org/10.5712/rbmfc8(29)828 DOI: http:// dx.doi.org/10.5712/rbmfc8(29)828

9. Castro RCL, Knauth DR, Harzheim E, Hauser L, Duncan BB. Avaliação da qualidade da atenção primária pelos profissionais de saúde: comparação entre diferentes tipos de serviços. Cad Saúde Pública. 2012;28(9):1772-84. DOI: http://dx.doi. org/10.1590/S0102-311X2012000900015

10. van Stralen CJ, Belisário SA, van Stralen TBS, Lima AMD, Massote AW, Oliveira CL. Percepção dos usuários e profissionais de saúde sobre atenção básica: comparação entre unidades com e sem saúde da família na Região Centro-Oeste do Brasil. Cad Saúde Pública. 2008;24(Suppl. 1):S148-58. DOI: http://dx. doi.org/10.1590/S0102-311X2008001300019

11. Sisson MC, Andrade SR, Giovanella L, Almeida PF, Fausto MCR, Souza CRP. Estratégia de Saúde da Família em Florianópolis: integração, coordenação e posição na rede assistencial. Saúde Soc. 2011;20(4):991-1004.

12. Araújo LUA, Gama ZAS, Nascimento FLA, Oliveira HFV, Azevedo WM, Almeida Júnior HJB. Avaliação da qualidade da atenção primária à saúde sob a perspectiva do idoso. Ciênc Saúde Coletiva. 2014;19(8):3521-32. DOI: http://dx.doi. org/10.1590/1413-81232014198.21862013

13. Harzeim E, Lima KM, Hauser L. Reforma da Atenção Primária à Saúde na cidade do Rio de Janeiro - avaliação dos três anos de Clínicas da Família. Pesquisa avaliativa sobre aspectos de implantação, estrutura, processo e resultados das Clínicas da Família na cidade do Rio de Janeiro. Porto Alegre: OPAS; 2013. [acesso 2017 Maio 5]. Disponível em: http:// www.sbmfc.org.br/media/file/reforma_atencao.pdf

14. Acurcio FA, Guimarães MDC. Acessibilidade de indivíduos infectados pelo HIV aos serviços de saúde: uma revisão de literatura. Cad Saúde Pública. 1996;12(2):233-42. DOI: http://dx.doi.org/10.1590/S0102-311X1996000200012

15. Travassos C, Martins M. Uma revisão sobre os conceitos de acesso e utilização de serviços de saúde. Cad Saúde Pública. 2004;20(Suppl. 2):S190-8. DOI: http://dx.doi.org/10.1590/S0102-311X2004000800014

16. Assis MMA, Jesus WLA. Acesso aos serviços de saúde: abordagens, conceitos, políticas e modelo de análise. Ciênc Saúde Coletiva. 2012;17(11):2865-75. DOI: http://dx.doi.org/10.1590/S1413-81232012001100002 
17. Domingues RCL, Amaral E, Bicudo-Zeferino AM. Conceito global: um método de avaliação de competência clínica. Rev Bras Educ Méd. 2009;33(1):148-51. DOI: http://dx.doi.org/10.1590/S0100-55022009000100019

18. Artmann E. O planejamento estratégico situacional no nível local: um instrumento a favor da visão multissetorial. Cadernos da Oficina Social 3: Série Desenvolvimento Local. Rio de Janeiro: Coppe/UFRJ; 2000.

19. Broeiro P. Justiça social e lei dos cuidados inversos. Rev Port Med Geral Fam. 2016;32(3):167-9.

20. Cantillon P. Teaching large groups. In: Cantillon P, Hutchinson L, Wood D, eds. ABC of learning and teaching in medicine. Londres: BMJ Publishing Group; 2003. p. 15-8. DOI: http://dx.doi.org/10.1136/bmj.326.7386.437

21. Murray M, Tantau C. Same-day appointments: exploding the access paradigm. Fam Pract Manag. 2000;7(8):45-50.

22. Murray M. Answers to your questions about same-day scheduling. Fam Pract Manag. 2005;12(3):59-64.

23. Ontario Ministry of Health and Long-Term Care. Health Quality Ontario. Advanced Access and Efficiency Workbook for Primary Care. London: Health Quality Ontario; 2011.

${ }^{a}$ Faculdade de Medicina de Valença - Fundação Dom André Arcoverde (FAA). Valença, RJ, Brasil. carol_reigada @yahoo.com.br (Autora correspondente) 